\title{
Estrategias educativas para la prevención de las infecciones de trasmisión sexual en jóvenes
}

\author{
Educational strategies for the prevention of sexually- \\ transmitted diseases in young people
}

\section{Bertha González Valcárcel ${ }^{\mathrm{I}}$; Maria Regla Bolaños Gutiérrez ${ }^{\mathrm{II}}$; Noria Liset Pupo Ávila ${ }^{\text {II }}$}

ILicenciada en Psicología y Pedagogía. Profesor Auxiliar. Escuela Nacional de Salud Pública. La Habana, Cuba.

${ }^{\mathrm{II}}$ Doctor en Medicina. Profesor Auxiliar. Universidad de La Habana. La Habana, Cuba.

\section{RESUMEN}

Introducción La epidemia del VIH/sida en Cuba, se caracteriza por un aumento lento y sostenido, con mayor incidencia en el último quinquenio y en jóvenes entre 15 a 29 años.

Objetivo Identificar las estrategias que se desarrollan en Cuba para la prevención de las infecciones de trasmisión sexual en los jóvenes.

Métodos Revisión documental y entrevistas en profundidad a los ejecutores de los programas nacionales.

Resultados Los programas dirigidos a los jóvenes estuvieron encaminados al desarrollo de conocimientos y comportamientos sexuales saludables. Las estrategias más empleadas fueron: educación, capacitación, consejería y campañas, se confeccionaron medios de apoyo: manuales, multimedia, juegos y accesorios. Existió sistematicidad en el desarrollo de las capacitaciones, sin embargo, no hubo adecuada permanencia de los consejeros y facilitadores una vez capacitados; no se comportó igual en los educadores pares y facilitadores de las escuelas. Los adolescentes encuestados tenían un buen nivel de conocimientos sobre las vías de transmisión y las formas de reducir el riesgo de contraer el VIH, sin embargo existió disonancia entre éstos y el uso sistemático del condón.

Conclusiones Los jóvenes cubanos son beneficiados por el Programa Nacional de Prevención y Control del VIH/sida que cuenta con un componente educativo y participación intersectorial que tributa a diferentes estrategias educativas. Se comprueba que a pesar de su buen nivel de conocimientos sobre el VIH/sida, estos 
jovenes no son sistemáticos en el uso del condón. Se propone que en las capacitaciones y actividades educativas se refuercen las técnicas de reflexión y análisis, considerando la sensibilidad de los adolescentes a la opinión de sus iguales y su influencia en el comportamiento.

Palabras clave: Estrategias educativas, infecciones de transmisión sexual, VIH/sida, jóvenes.

\begin{abstract}
Introduction The HIV/AIDS epidemic in Cuba shows a slow sustained rise, with higher incidence in 15-29 years-old young people in the last five years.

Objective To identify the strategies developed in Cuba for the prevention of sexually transmitted diseases in the young people.

Methods Documentary review and in-depth interviews made to those who implement the national programs.

Results The programs for young people were aimed at developing knowledge and healthy sexual behaviors. The most used strategies were education, training, counseling and campaigns; aid means were prepared such as manuals, multimedia, games and accessories. Training was systematic, however, advisers and facilitators, once trained, not always stayed in their posts, but the situation was not the same in peer educators and facilitators at schools. The surveyed teenagers exhibited good level of knowledge on ways of transmission and of reducing HIV risk. However, these pieces of knowledge did not influence the use of condom regularly. Conclusions The Cuban young people benefit from the National Program of HIV/AIDS Prevention and Control with educational orientation and intersectoral involvement, leading to several educational strategies. It was confirmed that despite their good level of knowledge on HIV/AIDS, the young people do not use condom on a regular basis. It is suggested that reflection and analysis techniques be emphasized in the training and education activities, taking into account that the young people are sensitive to the opinions of their peers and this may have an effect on their behaviors.
\end{abstract}

Key words: Educational strategies, sexually transmitted infections, HIV/AIDS, young people.

\title{
INTRODUCCIÓN
}

Las infecciones de transmisión sexual (ITS) constituyen riesgos graves para la salud y el bienestar de millones de personas en el mundo, y se encuentran entre las enfermedades infecciosas más comunes, además, facilitan la transmisión del virus de inmunodeficiencia humana (VIH), que causa el síndrome de inmunodeficiencia adquirida (sida); el que se ha convertido sin dudas en uno de los principales problemas de la salud en la actualidad, con una importante morbilidad y mortalidad en los sectores más jóvenes de la población, por consiguiente, produce cuantiosos costos sociales y pérdidas de vidas. ${ }^{1,2}$ Esta enfermedad no distingue edad, sexo, raza, ocupación, país, condición social, etnia, nivel socioeconómico o religión. ${ }^{3}$ 
Actualmente, en el mundo viven con el VIH alrededor de 38,6 millones de personas; 2,6 millones más que en el $2004,{ }^{4}$ de ellos 10,3 millones son jóvenes de 15 a 24 años lo que representa el $42 \%$ de las personas infectadas recientemente. Por otra parte, el $50 \%$ de las nuevas infecciones por el VIH (casi seis mil diarias) sucede en personas jóvenes. Si se hace un análisis de género, se aprecia que infecta a un número cada vez mayor de mujeres, y a edades significativamente más tempranas que en el caso de los varones (PNUD/FM/cub/03/01/18/A/01/99. Fortalecimiento de la respuesta nacional multisectorial para la prevención y atención de la epidemia del VIH/SIDA en la República de Cuba. Julio 2003).

Cuba se considera entre los países caribeños menos afectados. La epidemia se ha caracterizado por un aumento lento y sostenido con mayor incidencia en el último quinquenio. Las estadísticas de la Dirección Nacional de Epidemiología a finales del 2007 mostraron un incremento en el número de casos en relación con el año precedente. Se han diagnosticado un total acumulado, desde 1986, de 9304 seropositivos, $80 \%$ de estos son del sexo masculino y el $20 \%$ mujeres. Los hombres que tienen sexo con otros hombres constituyen el grupo de mayor vulnerabilidad y el $86 \%$ de los casos masculinos reportados. ${ }^{5}$ La forma de infección predominante es la transmisión sexual.

La capital de la Isla es la más afectada por la epidemia, con el 53,3\% de los casos del país, todos sus municipios tienen estimados de prevalencia superior a la media nacional.

A lo largo de la historia de la aparición del virus en Cuba, la incidencia de la infección se ha registrado fundamentalmente en edades entre 15 a 29 años, grupo donde se concentra algo más del $70 \%$, aunque en los últimos años ha aumentado en edades más avanzadas, entre 25 a 34 años. ${ }^{5}$ De ahí la importancia de dirigir los esfuerzos a la educación de la sexualidad para la prevención de la infección.

La Organización Mundial de la Salud (OMS) considera a la adolescencia como la etapa de la vida que se desarrolla entre los 10 y 19 años de edad, con dos fases en ella: la adolescencia temprana de 10 a 14 años y la adolescencia tardía de 15 a 19 años. En esta etapa se produce una interacción de los procesos de desarrollo biológico, psicológico y social en las personas que conducen a la madurez, están conformándose la identidad, la autonomía y el proyecto de vida. ${ }^{6}$

En esta época es común la curiosidad e interés sobre temas relacionados con la sexualidad, sobre todo lo relacionado con el aspecto erótico. Lo anterior implica la necesidad de un abordaje integral de la sexualidad donde juega un papel determinante la promoción de comportamientos responsables desde edades tempranas, encaminados a transformar positivamente las situaciones sociales que generan riesgos para la salud.

Se sabe que existen algunos factores que hacen particularmente vulnerables a los jóvenes a la infección por VIH/sida y otras ITS. En este sentido, el inicio de las relaciones sexuales en edades precoces significa que se tendrán mayor número de parejas y, por lo tanto, una mayor exposición a infecciones, que los individuos que retrasan el inicio de la actividad sexual hasta la adolescencia tardía o adultez joven. Los varones adolescentes son menos estables y efectivos en el uso de anticonceptivos que los adultos, por lo que se benefician menos de su efecto protector, tienen menos parejas estables, se refugian en múltiples parejas sexuales y de hecho aumenta la exposición a las infecciones, además que, el deseo de aventura y el afán por explorar cosas nuevas, predispone a los jóvenes a adoptar comportamientos de riesgo en relación con el VIH. ${ }^{7}$ 
La influencia que pueden ejercer los distintos espacios donde los adolescentes se desarrollan en su vida cotidiana es determinante, más aun si se considera que en esta etapa de la vida, las relaciones con sus coetáneos revisten un carácter especial. El presente estudio tiene como objetivo identificar algunas estrategias educativas que se desarrollan en Cuba para la prevención de las ITS y el VIH en los jóvenes.

\section{MÉTODOS}

Se realizó una revisión bibliográfica y documental sobre las estrategias educativas realizadas por diferentes instituciones, entre ellos, el Centro Nacional de Prevención de las ITS/ VIH/sida del Ministerio de Salud Pública (MINSAP), el departamento de salud escolar del Ministerio de Educación (MINED) y el Centro Nacional de Educación Sexual (CENESEX). Se realizaron además, entrevistas a profundidad a los responsables de la línea jóvenes y asesores de salud para identificar las estrategias educativas que se ejecutan en la preveción de las ITS/VIH/sida en los jóvenes.

\section{RESULTADOS y DISCUSIÓN}

\section{Implementación y desarrollo inicial de acciones contra el VIH/sida}

Se pudo comprobar que desde 1986, cuando se diagnosticaron los primeros casos de infección por el VIH en el país, se implementaron, desarrollaron y fortalecieron, numerosas acciones encaminadas a limitar la progresión de la epidemia y minimizar su impacto en la población cubana, donde fue decisiva la voluntad política. De esta manera, se creó en la primera mitad de los ochentas, el Grupo Operativo para el Enfrentamiento y Lucha contra el sida (GOPELS), constituida como la autoridad nacional para la coordinación y conducción del abordaje multisectorial de la epidemia, dirigida por el Gobierno e integrada por todos los organismos y organizaciones que participan en el Plan Intersectorial. Este grupo se perfeccionó en 1997 con un enfoque más integral mediante el Plan Intersectorial para la Educación, Prevención y Control de las ITS/VIH/sida. Abarca todos los municipios y provincias del país, para potenciar la respuesta a nivel local, desarrollar capacidades y fortalecer las acciones en los territorios más afectados por el VIH.

El Programa Nacional para la Prevención y Control del VIH/sida, se implementó desde 1986, integra los componentes de educación, prevención, diagnóstico, vigilancia epidemiológica, atención y cuidados e investigaciones. Su componente educativo tiene el propósito de promover conductas sexuales, actitudes y prácticas saludables en la población, con énfasis en los grupos más afectados por la epidemia, a través de la participación comunitaria y la intersectorialidad.

Este programa se orienta hacia el enfrentamiento de la epidemia desde todos los sectores del quehacer nacional y tiene en cuenta factores condicionantes como los psicológicos, los culturales y los sociales, donde se involucra a la familia, la escuela y los medios de comunicación social. Estos resultan de gran importancia por su contribución a la formación de normas, valores y modelos de conductas que pueden facilitar o entorpecer comportamientos sexuales saludables, en consistencia con la pretensión de una planificación hacia un futuro deseado y el logro de una respuesta ampliada, con la visibilidad de cada sector en relación con la situacióna nacional. ${ }^{8,9}$ 
(Ochoa SR, Pérez PF, Valdés T JR. Manual de planificación estratégica para los sectores. PNUD. Grupo Nacional de las ITS/VIH/sida, MINSAP. Cuba, pag. 7-8).

\section{Acciones de fortalecimiento}

Como respuesta nacional al comportamiento del VIH, se creó el Plan Estratégico Nacional para las ITS/VIH/sida 2001-2006, en el que se formula la respuesta multisectorial de promoción de la salud, prevención de la enfermedad, atención sanitaria y social en la población general y de riesgo, con el objetivo de "...limitar la progresión de la epidemia y minimizar su impacto en la población cubana". ${ }^{9}$

De esta manera el MINSAP, a través del Centro Nacional de Prevención de las ITS/VIH/ sida (CNP), el Misterio de Educaciçon (MINED), mediante el Departamento de Salud Escolar y el Centro Nacional de Educación Sexual (CENESEX), junto a otros sectores, planifican y desarrollan programas y proyectos educativos, apoyados por organismos y organizaciones nacionales e internacionales.

Estos programas están dirigidos al desarrollo de conocimientos y comportamientos adecuados en los adolescentes, para que puedan vivir una sexualidad sana, con un disfrute pleno, pero teniendo en cuenta que el VIH existe y "el riesgo de contraerlo está entre nosotros" lo importante es saber como evitarlo (Ochoa SR, Pérez PF, Valdés T JR. Manual de planificación estratégica para los sectores. PNUD. Grupo Nacional de las ITS/VIH/sida, MINSAP. Cuba, pag. 7-8).

Entre las estrategias empleadas se encuentran: la información, la comunicación, la educación, la capacitación, la consejería y las campañas. Para ellas se confeccionan los programas, con el apoyo de diferentes materiales escritos, visuales y audiovisuales que permiten la promoción y el apoyo a las estrategias mencionadas. Entre ellas se encuentran: los afiches, plegables, vallas, manuales, videos, revistas, multimedias, entre otros.

La consejería es una herramienta de información y comunicación por excelencia, resulta ser una forma eficiente de producir conocimientos y mediante ella se puede prevenir la propagación de las ITS y el VIH. Es una forma profesional de brindar ayuda, si se tiene en cuenta que el joven puede expresar sus sentimientos, sus miedos y temores, y generar alternativas adecuadas de comportamientos y la percepción de la autoeficacia. Existen diferentes formas de consejería: telefónica, anónima y cara a cara.

La consejería Telefónica nacional, conocida como LINEAYUDA, es anónima y confidencial. Tiene alcance nacional y permite que las personas se comuniquen desde cualquier parte del país a través de una línea telefónica para obtener información y orientación sobre el tema de las infecciones de transmisión sexual y el VIH, también existe este servicio en otras provincias del país. La consejerìa Anónima es otro servicio de orientación e información sin identificación personal. Se encuentra a nivel provincial y se han creado condiciones en algunos municipios sobre todo en Ciudad de la Habana para su funcionamiento. Las consejería Cara a Cara se realizan a nivel nacional, provincial, municipal y por áreas de salud, en estos últimos años se ha logrado un mayor alcance, lo que ha permitido a la población tener la información y orientación necesarias para realizarse el estudio diagnóstico del VIH y otras ITS. 7,10

Las capacitaciones van encaminadas a la formación de los promotores de salud, que se dedican a la educación de pares en las escuelas, centros de trabajo y comunidad. También para formar a los consejeros, entre los que se encuentran jóvenes con características determinadas, maestros, estudiantes, entre otros; la 
modalidad empleada para su desarrollo es el taller, impartido por un personal calificado, con una metodología activa, participativa y en forma de cascada, desde el nivel nacional hasta el municipal. Esto permite una mejor cobertura y participación al extender las capacitaciones de forma organizada a todo el país.

Entre las metodologías más empleadas se encuentran la planteada por Paulo Freire, basada en el principio de acción-reflexión-acción y la investigación acción participativa, basada en las relaciones del pensamiento, los sentimientos y la acción, como fuerza movilizadora de la conducta de aprendizaje subjetivo, representada por Kart Lewis y sus seguidores. ${ }^{7}$

El taller es la figura docente más utilizada, ya que tiene como función fundamental la reflexión colectiva de problemas y sus posibles soluciones, lo que contribuye al desarrollo de habilidades para el trabajo en grupos, a la integración de conocimientos y crea conciencia en los adolescentes para que mantengan una conducta adecuada en cuanto a su pareja y su salud. Se realizan de múltiples maneras: dialogadas, reflexivas, de tácticas de capacitación, educación, comunicación, sensibilización e intersectorial, con posibilidades de vincular vivencias y testimonios de personas portadoras del virus, a su vez incorpora problemas sociales relacionados con la sexualidad, particularmente las ITS y el VIH.

Como apoyo a la docencia se confeccionaron medios que facilitan el proceso educativo: manuales, folletos, multimedias, juegos, entre otros. Están dirigidos a adolescentes, estudiantes, docentes y a la familia. Constituyen una herramienta valiosa en la docencia y también contribuyen a la cultura general integral y a la preparación para la vida. ${ }^{7,11-16}$ (Sanchez FJ, Duque SI, Coll SG. Manual de capacitación para facilitadores juveniles en educación sobre las ITS/VIH/sida. CNP de las ITS/VIH/sida. La Habana: MINSAP. p.7-9. Saber si da victoria. Guia de preguntas y respuestas para el juego didactico. Proyecto dirigido a jovenes y adolescentes MINSAP. PNUD).

Otra de las estrategias empleadas son las Campañas para la prevención de VIH, que en cada año se planifican con la participación de diferentes sectores sociales, entre las que se encuentran: La Campaña de Verano y la Campaña por el Día Mundial de lucha contra el sida. Durante ellas se realizan diversas actividades dirigidas fundamentalmente a los adolescentes. Se utilizan diferentes medios y formas de comunicación como son: eslogan, spot televisivo, afiche, vallas, llaveros, pull-overs, entre otros, con el fin de promocionar y lograr la participación en estas actividades.

\section{Efectividad de las actividades desarrolladas}

Se pudo constatar que existe sistematicidad en el desarrollo de las capacitaciones según las entrevistas realizadas y la revisión efectuada a los documentos de control del proceso capacitante. Sin embargo, no existe una permanencia de los consejeros y facilitadores una vez capacitados, por lo que es necesario investigar los motivos que inciden en estas deserciones para encontrar mecanismos de incentivos a esta actividad que propicien la estabilidad en el trabajo del personal capacitado.

No sucede lo mismo con los educadores pares y facilitadores del MINED quienes plantearon que no existen grandes dificultades, pues al graduarse, los estudiantes siempre van a trabajar como docentes en un aula donde aplican los conocimientos y actividades aprendidas. Por otra parte, la educación sexual constituye desde hace muchos años, un eje transversal de la educación de manera que se brindan temas de sexualidad a partir de la vía curricular y la extracurricular en todos los niveles de 
enseñanza; esto permite que los estudiantes reciban de forma sistemática una adecuada educación sexual relacionada con su edad.

Estudios realizados en jóvenes en Cuba, han revelado que poseen un buen nivel de conocimientos sobre las vías de transmisión de la ITS y las formas de reducir el riesgo de contraer el VIH mediante el uso del condón en cualquier tipo de relación. Para los jóvenes, las principales fuentes de información sobre VIH fueron: los familiares, los amigos y la escuela, seguida por la TV y la radio con menos cuantía mediante las Campañas. Existe inconsistencia entre el conocimiento y el comportamiento, al expresar que estas fuentes de información han tenido influencia en su conducta sexual y sin embargo se constató que aproximadamente el $50 \%$ o más de los adolescentes de la enseñanza media superior y del curso de superación integral, refieren usar condón solo ocasionalmente tanto en las relaciones sexuales estables como no estables ${ }^{6}$ (Estrada A. ME, González VB, Pérez PJ, Zaldivar DI. Estrategia de Comunicación Educativa en VIH a jóvenes del Curso de Superación Integral. Florida Camagüey, 2006-2007).

Todo ello pone de manifiesto la disonancia entre el conocimiento que poseen los adolescentes sobre el uso del condón y el comportamiento inadecuando con respecto a su uso sistemático en las relaciones sexuales, lo que pudiera estar condicionado por las características propias de esta etapa de la vida, donde muchos comportamientos están en fase de conformación, entre ellos, los que corresponden al área de la sexualidad. Esto constituye un aspecto de especial importancia como parte del proyecto de vida de cada joven, que se va estableciendo en sus formas de manifestar las organizaciones valorativas, conocimientos, creencias y actitudes de forma estable en su vida sexual.

En este proceso los maestros y educadores pares son una fortaleza, siempre que apliquen las herramientas aprendidas para poder hacerlo, por lo que la escuela constituye un espacio favorable para la formación de comportamientos sexuales saludables en esta etapa de la vida. Los resultados apuntan sin dudas hacia agentes socializadores sobre los cuales deben reforzarse también las diferentes acciones y la escuela constituye una fuerte potencialidad en este sentido.

Sería oportuno reforzar las técnicas de reflexión y análisis, en las capacitaciones de los educadores pares, si se considera que la mayoría de los adolescentes son extremadamente sensibles a la opinión de sus iguales y que la percepción de lo que piensan los compañeros tiene por lo común mayor influencia en el comportamiento sexual o en cualquier otro tipo de comportamiento arriesgado. ${ }^{16}$ Se ha de tener en cuenta la elevada representación que alcanzan los amigos y los familiares en ese estudio siendo esto de suma importancia para lograr una mejor sensibilización hacia comportamientos seguros en los adolescentes.

Algunos adultos opinan que enseñar a los adolescentes cuestiones relacionadas con el sexo y la sexualidad les animará a realizar actividades sexuales demasiado temprano, sin embargo, los estudios de ONUSIDA, han indicado que esta creencia es errónea, y plantean que:

...de hecho, cuando ellos reciben información precisa sobre la sexualidad y el VIH, hay más posibilidades de que retrasen sus actividades sexuales y utilicen preservativos cuando finalmente decidan tener una relación sexual. ${ }^{17}$

Cevallos $^{18}$ en su artículo sobre la ignorancia y la mala o contradictoria información de los jóvenes en muchos países de América Latina, esboza que los jóvenes cubanos poseen informaciones adecuadas provenientes de diversas fuentes que facilitan o refuerzan el conocimiento sobre la sexualidad, y de la necesidad de esa 
información ante la presencia generalizada de una sexualidad activa en los adolescentes. Mabel Bianco, citada por Cevallos, ${ }^{18}$ dice que:

Hay que enfrentar la realidad, los jóvenes van a tener sexo con o sin la información que les demos, y por eso hay que darles información e instrumentos para que tomen las decisiones oportunas.

Finalmente se puede concluir que los adolescentes cubanos son beneficiados por un Programa Nacional de Prevención y Control del VIH/sida. Este programa cuenta con un importante componente educativo y participación intersectorial, que da salida de manera apropiada y sistemática a diferentes estrategias educativas con métodos, técnicas y medios que facilitan el conocimiento de los adolescentes sobre este problema. Existen dificultades en la permanencia de los educadores pares y consejeros formados, lo que puede estar indicando la necesidad de reforzar los mecanismos de incentivos que permitan mayor motivación por la labor educativa que realizan en el desarrollo de la autoestima y la asertividad en los adolescentes para la toma de decisiones de comportamientos sexuales seguros y saludables. Se considera a la escuela como un lugar favorable para la educación sexual de los adolescentes aunque existen otros espacios que ellos ocupan en la sociedad que son importantes también para descubrir y entender aquellos valores más íntimos que guían la actitud y actividad individual en esta importante etapa de la vida.

\section{REFERENCIAS BIBLIOGRÁFICAS}

1. Programa Conjunto de las Naciones Unidas sobre el VIH/SIDA. Asamblea General de las Naciones Unidas sobre el VIH/SIDA. Resumen de la Declaración de compromiso en la lucha contra el VIH/SIDA, jun. 25-27. Nueva York: ONUSIDA; 2001.

2. Los jóvenes y el SIDA. ¿Podemos evitar una catástrofe? [sitio en Internet]. 2002 [citado 22 Feb 2007]. Disponible en:

http://info.k4health.org/pr/prs/sl12edsum.shtml

3. ONUSIDA. Período extraordinario de sesiones de la Asamblea General de las Naciones Unidas sobre el VIH/SIDA, 25-27 junio. Declaración de compromiso en la lucha contra el VIH/SIDA. Nueva York: ONUSIDA; 2001.

4. ONUSIDA. Resumen de Orientación. Informe sobre la epidemia mundial del sida 2006 [sitio en Internet]. 2007 Ene [citado 8 Ene 2007] Disponible en:

http://data.unaids.org/pub/GlobalReport/2006/2006 GR CH02 es.pdf

5. Anuario Estadístico de Salud. La Habana: MINSAP; 2006.

6. González Valcárcel B, Núñez Aragón E, Couturejuzon González L, Amable Ambrós

Z. Conocimientos y comportamientos sobre el VIH/sida en adolescentes de enseñanza media superior. Rev Cubana Salud Pública [serie en Internet]. 2008 [citado 8 Nov 2009];34(2). Disponible en:

http://scielo.sld.cu/scielo.php?script=sci arttext\&pid=S0864-

34662008000200006\&lng=es

7. Ochoa Soto R, Sánchez Fuentes J, Villalón M, Duque Santana I, Hernández Fernández M, Cachón Asusta L, Pérez Pérez F. Manual para médicos de Familia sobre ITS VI sida. La Habana: MINSAP/PNUD; 2003. 
8. Programa Nacional de Control y prevención del VIH/SIDA. La Habana: MINSAP; 1997.

9. Plan estratégico Nacional ITS/VIH/SIDA 2001-2006. Resolución ministerial 110. República de Cuba. La Habana: MINSAP; 2001.

10. Multimedia sobre consejería. Cuba consejería. Centro Nacional de prevención de las ITS/VIH/sida. La Habana: MINSAP/PNUD/ONUSIDA; 2006.

11. Castro Alegret PL, Torres Cueto MA, López Gómez AB, González Hernández A, Padrón Echevarria AR, Baxter Pérez $E$, et al. Vivir nuestra sexualidad y prevenir el VIH sida. . La Habana: MINED/UNFPA/PNUD (Ediciones Molinos Trade S. A);2004.

12. Castro Alegret PL, Torres Cueto MA, López Gómez AB, González Hernández A, Padrón Echevarria AR. Conocer nuestra sexualidad y prevenir el VIH/sida. Para estudiantes y adolescentes. La Habana: MINED/PNUD (Ediciones Molinos Trade S. A); 2004.

13. Castro Alegret PL, Padrón Echevarria AR, García González A. Familia, sexualidad y educación. Serie Educación y sexualidad. Proyecto Educación formal para una conducta sexual responsable. La Habana: Programa Nacional de educación sexual, MINED; 2003.

14. Sánchez Fuentes J, Duque Santana I, González Hernández A. Manual de capacitación para facilitadores juveniles en educación sobre las ITS/VIH/sida. Centro Nacional de prevención de las ITS/VIH/sida. La Habana: MINSAP; 2006.

15. Acevedo García I, Álvarez Ramírez S, Cancio Enríquez I, Hernández Pesans O, Duque Santana I. La mujer en la prevención del sida. De mujer a mujer. Manual para promotoras de salud. LA Habana: MINSAP; 2004.

16. López Brito M. VIH/SIDA/ITS. Una perspectiva de los adolescentes. Instituto Universitario a.c. Colima, col. México. Foro SIDA 2003. La Habana: Palacio de las Convenciones; 2003.

17. ONUSIDA. Programas Nacional de SIDA /00.17E. Guía para el monitoreo y evaluación. Ginebra: ONUSIDA; 2000.

18. Cevallos D. Jóvenes atrapados entre el sida y la ignorancia. Artículo producido por: Inter Press Service News Agency (IPS) con motivo del 1 de diciembre 2002. Día Mundial de la Lucha Contra el Sida [sitio en Internet]. [Ene 2007]. Disponible en: http://www.scielosp.org/pdf/rcsp/v34n2/v34n2a06.pdf

Recibido: 8 de diciembre de 2009.

Aprobado: 5 marzo de 2010. 
Bertha González Valcárcel. Escuela Nacional de Salud Pública. Calle Línea esq. a I. El Vedado 10400. La Habana, Cuba. E-mail: berthag@infomed.sld.cu 